\title{
openheart Exercise-based cardiac rehabilitation after heart valve surgery: cost analysis of healthcare use and sick leave
}

\author{
T B Hansen, ${ }^{1,2,3}$ A D Zwisler, ${ }^{3,4,5}$ S K Berg, ${ }^{3} \mathrm{~K}$ L Sibilitz, ${ }^{3}$ L C Thygesen, ${ }^{4}$ \\ P Doherty, ${ }^{6}$ R Søgaard ${ }^{7,8}$
}

To cite: Hansen TB, Zwisler AD, Berg SK, et al. Exercise-based cardiac rehabilitation after heart valve surgery: cost analysis of healthcare use and sick leave. Open Heart 2015;2:e000288. doi:10.1136/openhrt-2015000288

Received 28 April 2015 Revised 16 June 2015 Accepted 22 June 2015

\section{(DrossMark}

For numbered affiliations see end of article.

Correspondence to

TB Hansen;

tbh@regionsjaelland.dk

\section{ABSTRACT}

Background: Owing to a lack of evidence, patients undergoing heart valve surgery have been offered exercise-based cardiac rehabilitation (CR) since 2009 based on recommendations for patients with ischaemic heart disease in Denmark. The aim of this study was to investigate the impact of $\mathrm{CR}$ on the costs of healthcare use and sick leave among heart valve surgery patients over 12 months post surgery.

Methods: We conducted a nationwide survey on the CR participation of all patients having undergone valve surgery between 1 January 2011 and 30 June 2011 $(n=667)$. Among the responders $(n=500,75 \%)$, the resource use categories of primary and secondary healthcare, prescription medication and sick leave were analysed for CR participants $(\mathrm{n}=277)$ and nonparticipants ( $n=223$ ) over 12 months. A difference-indifference analysis was undertaken. All estimates were presented as the means per patient $(95 \% \mathrm{Cl})$ based on non-parametric bootstrapping of SEs.

Results: Total costs during the 12 months following surgery were $€ 16065$ per patient $(95 \% \mathrm{Cl} 13730$ to 18 399) in the CR group and $€ 15182$ (12 695 to 17670 ) in the non-CR group. CR led to 5.6 (2.9 to $8.3, p<0.01)$ more outpatient visits per patient. No statistically significant differences in other cost categories or total costs $€ 1330$ ( -4427 to 7086 , $\mathrm{p}=0.65$ ) were found between the groups.

Conclusions: $\mathrm{CR}$, as provided in Denmark, can be considered cost neutral. CR is associated with more outpatient visits, but CR participation potentially offsets more expensive outpatient visits. Further studies should investigate the benefits of $C R$ to heart valve surgery patients as part of a formal cost-utility analysis.

\section{INTRODUCTION}

With an ageing population, heart valve disease incidence and number of heart valve procedures performed are both increasing, ${ }^{1}$ but the associated economic burden is sparsely investigated. ${ }^{2}$ Additionally, over the past 15 years, the survival and management of patients with cardiovascular disease has improved substantially, resulting in a more complex case profile by the time of heart

\section{KEY QUESTIONS}

What is already known about this subject? Even though cardiac rehabilitation is recommended after heart valve surgery, there is a lack of studies investigating the economic implications. Several studies have highlighted the difficulties of implementing results from randomised trials due to structural differences in settings and large variation in clinical practice including referral, participation and adherence rates, among other substantial factors.

What does this study add?

This study examines the cost consequences of offering cardiac rehabilitation in a national healthcare system with referral rates, participation rates and programme variation of a real-life setting using multiple data sources. We find that more than half of patients in our sample choose to participate in rehabilitation and that participation is overall cost neutral to the healthcare system due to provision costs being outweighed by less costly service use elsewhere.

How might this impact on clinical practice? While randomised controlled trials with economic evaluation conducted alongside remain important sources for informing future priorities. However, this study provides important reference values for how heart valve patients value rehabilitation and what consequences universal provision has elsewhere in a healthcare system and at the labour market.

valve surgery, ${ }^{3}$ which may impact the recovery path and healthcare use following surgery.

Although health-related quality of life (HRQL) improves following heart valve surgery, ${ }^{4}$ difficulties resuming normalcy and physical function, along with anxiety and depression, can persist. ${ }^{5}$

A recent Cochrane systematic review on exercise-based cardiac rehabilitation (CR) to patients after heart valve surgery identified only two randomized trials including a total of 148 patients. Both trials showed 
improvements in exercise capacity in favour of CR compared to usual care, but the evidence was inadequate regarding other outcome measures, such as mortality and HRQL. ${ }^{6}$ Due to the lack of evidence, CR has been offered nationwide to heart valve surgery patients in the Danish health care system since 2009 based on recommendations for patients with ischemic heart disease ${ }^{78}$ for which CR is shown to reduce hospital admissions, improve HRQL and, to some extent, reduce total and cardiovascular mortality. ${ }^{9}$ Studies are inconsistent on whether CR participation impacts the rate of return to work and number of days off work due to sick leave. ${ }^{10} 11$

Two systematic reviews including economic evaluations of $\mathrm{CR}$ of varying quality have concluded, that $\mathrm{CR}$ is cost effective among patients with myocardial infarction, coronary artery bypass surgery, percutaneous transluminal angioplasty and heart failure ${ }^{12}$ despite heterogeneity across studies. No studies have assessed the effect of CR to heart valve surgery patients from an economic perspective. Findings from the existing economic evaluations may not be applicable to heart valve surgery patients, since these may have a different cost profile, preferences and benefit differently from CR participation. Although the beneficial effects of CR have been demonstrated, participation and adherence remain sub-optimal, ${ }^{14}$ which may also complicate transfer of findings to real-life settings from economic evaluations based on the limited number of randomized trials, which cannot be pooled. ${ }^{12}$ may not be easily transferred to real-life settings.

The objective of this study was to investigate the impact of CR, after heart valve surgery, on the costs associated with healthcare use and sick leave among consecutive patients, based on national practice.

\section{MATERIALS AND METHODS Study design}

This was a 12-month follow-up of a prospective comparative observational study of CR participation versus nonparticipation using data acquired from several registers and merged at the individual level using a Statistics Denmark server. Information on CR participation was derived from a nationwide survey.

\section{Population}

A cohort of consecutive patients undergoing heart valve surgery in Denmark between 1 January 2011 and 30 June 2011 , and $18+$ years of age and not undergoing percutaneous valve replacement, was identified in the National Patient Register ${ }^{15}$ using the Danish SKS procedure codes based on the NOMESCO (Nordic Medico-Statistical Committee) classification of Surgical Procedures: KFG (tricuspid valve surgery), KFJE and KFJF (pulmonary valve surgery), KFK (mitral valve surgery) and KFM (aortic valve surgery) $(n=770)$. Of these, 51 patients had a research-protected address and 52 died before December 2011. We invited the remaining 667 patients to participate in a nationwide postal survey 6-12 months post surgery on CR participation. The questions on CR participation, which also included a definition of CR specifying the elements of a CR programme, have been developed and previously tested by the Danish Heart Association to assess CR participation among patients with ischemic heart disease. ${ }^{16}$ A reminder was sent after two weeks, and the response rate was 500/667 (75\%).

\section{Cardiac rehabilitation in Denmark}

CR recommendations in Denmark include physical exercise training, psychosocial support, disease education, diet counselling, smoking cessation sessions and follow-up of medical treatment and CR initiation approximately 1-2 weeks after surgery and 4-6 weeks for physical exercise training due to sternal wound recovery. ${ }^{7} \quad 8 \quad 17$ CR programmes are usually provided in outpatient settings in the hospitals. However, since 2007, municipalities in Denmark have also been able to provide CR based on the same recommendations either fully or as a shared programme with a hospital. At the time of the survey, 32/34 hospitals had a CR programme, and among the 98 municipalities, 11 had a full programme, whereas 30 municipalities participated in a shared programme with a hospital. The hospital-based programmes lasted 5-24 weeks (mean 11.5). In the municipalities, the programmes lasted 2-12 weeks (mean 8.5). Most exercise training sessions across programmes were conducted twice weekly. ${ }^{18}$

Of the 500 patients, 277 (55\%) patients reported to have participated in CR versus 223 (45\%) not participating. Non participation was due to patients not being referred (18\%) and to being referred but choosing not to participate $(27 \%)$. The main self-reported reasons for non-participation were readmissions, comorbidity, having returned to work, transportation and being in physical good shape and considering CR unnecessary.

CR participants reported to have received physical training $(277 / 277,100 \%)$, formal psychosocial support $(81 / 277,29 \%)$, disease education $(144 / 277,52 \%)$, diet counselling $(169 / 277,61 \%)$ and follow-up of medical treatment $(219 / 277,79 \%)$ as part of the CR programme. Among smokers 14/48 (29\%) had attended smoking cessation sessions". Only patients, who had participated in a formal programme in either a hospital or a municipality, were included as CR participants. Among these, 230 of the 277 patients reported following a full or shared hospital-based CR programme.

\section{Baseline variables}

For baseline assessment, we obtained information from several registers at Statistics Denmark registers. Preoperative and postoperative comorbidity was calculated using the index described by Charlson et $a l^{19}$ for each patient using the primary and secondary diagnosis for hospital contacts.

\section{Resource use and costs}

The cost analysis included the categories: primary healthcare, secondary healthcare (hospital-based inpatient and 
outpatient visits), prescription medication and production loss due to sick leave. All costs were inflated to $2012-€$ using the general consumer price index and a currency conversion rate of $745 \mathrm{DKK}=100 €$. Costs associated with hospital-based CR were included in the cost parameter of outpatient visits.

\section{Measurement and valuation of healthcare use Primary healthcare}

All contacts in the primary healthcare sector are recorded in the Danish National Health Service Register for Primary Care. ${ }^{20}$ We categorised service providers into four groups: general practitioners (codes 80-84, 89), medical specialists (codes 1-21, 23-24), physiotherapists (codes 51, 62) and psychologists (code 63). Resource use in primary healthcare was valued using the tariffs of national agreements between the professional associations of medical specialists and the Danish National Health Service.

\section{Secondary healthcare}

From the National Patient Register ${ }^{15}$ we obtained information on hospital admissions and outpatient visits. Resource use in secondary healthcare was valued using the tariffs of the case-mix system of diagnosis-related groupings (DRG) for inpatient services and the Danish Ambulatory Grouping System (DAGS) for outpatient visits.

Inpatient admissions were divided into acute and nonacute admissions in departments of cardiology, thoracic surgery and other departments. Primary diagnoses were manually verified for the admissions during the first month postsurgery. Outpatient visits were divided into cardiology, thoracic surgery and other departments.

\section{Prescription medication}

The Danish National Prescription Register includes data on prescription medication dispensed in Denmark. ${ }^{21}$

Medications of particular relevance were identified using the Anatomical Therapeutic Chemical (ATC) classification codes: ACE inhibitors (C09A, C09B), $\beta$-blockers (C07), spironolactone (C03DA01), lipid-modifying agents (C10A, C10B), digoxin (C01AA05), diuretics (C03A, C03C), antithrombotic agents except anticoagulant treatment (B01A), anticoagulant treatment (B01AA, B01AE07, B01AF02, B01AF01), psycholeptics (N05A), antiarrhythmic drugs (C01BD01, C01BD07) and analgaesics (M02, N02). Medication use per patient was calculated using the defined daily dose (DDD), which is the assumed average maintenance dose per day of a drug used for its main indication, ${ }^{22}$ and valued by the retail price. Records without DDD values, respectively 810/ 25,693 and $997 / 33,715$, presurgery and postsurgery, were manually reviewed and omitted from the analysis due to low impact on the results.

\section{Sick leave}

Information on sick leave was acquired from the DREAM database including weekly information on social benefits, which is administered by the Danish
Ministry of Employment. Only sick leave lasting for more than 2 weeks is registered in the DREAM database. ${ }^{10} 23$ Productivity losses due to sick leave were based on national age-matched and gender-matched gross wages.

\section{Statistical methods}

Data were collected 1 year before and 1 year postsurgery, which allowed for the analysis of (1) within-group differences over time (costs incurred the year before discharge minus the costs incurred the year after discharge) and (2) differences between within-group differences (CR vs no CR). The purpose of this analytical approach is to adjust for endogenous variables that do not vary over time (eg, patient characteristics) and exogenous variables that do not vary between groups (eg, context of rehabilitation).

Baseline characteristics were analysed using Student $t$ tests for continuous variables and $\chi^{2}$ tests for categorical variables.

Healthcare utilisation, sick leave and costs were reported as mean resource use and cost per patient as well as the differences between them. Non-parametric bootstrapping with 5000 replications was used to estimate the SEs and 95\% CIs due to the usual nature of resource-use data, which are right-skewed. ${ }^{24}$ There was no loss to follow-up.

Statistical analyses were carried out using Stata V.13. A $p$ value below 0.05 was considered statistically significant.

\section{ETHICS}

The study was approved by the Danish Data Protection Agency (2011-41-6378, 2013-41-1643), and the data were handled in accordance with the Act on Processing of Personal Data. Non-interventional studies do not require approval from ethics committees in Denmark.

\section{RESULTS}

Baseline characteristics are described in table 1, with minor differences between the $\mathrm{CR}$ and no $\mathrm{CR}$ groups according to age, marital status and type of surgery.

A statistically significant difference in the mean number of total outpatient visits per patient between groups was observed, with a higher number of 5.6 (95\% CI 2.9 to 8.3, $\mathrm{p}<0.01$ ) visits in the CR group (table 2). The difference in the total number of outpatient visits between groups was reflected in a non-statistically significant difference in costs, with higher costs per patient in the CR group of EUR $407(-378 ; 1,191, p=0.29)$ (table 3$)$. Of the total number of outpatient visits in the CR group, 8.6 (7.5 to 9.6) visits per patient were due to physical exercise training as part of the CR programme (data not shown). The difference in the total number of outpatient visits between groups was reflected in a non-statistically significant difference in costs, with higher costs per patient in the CR group of $€ 407$ ( -378 to $1191, \mathrm{p}=0.29$; table 3 ).

Except for differences in the number of outpatient visits, we found no statistically significant differences in 
Table 1 Baseline characteristics of consecutive patients eligible for exercise-based cardiac rehabilitation after heart valve surgery

\begin{tabular}{|c|c|c|c|c|c|c|c|}
\hline & $\begin{array}{l}\text { Total } \\
\text { population } \\
(n=667)\end{array}$ & $\begin{array}{l}\text { Responders } \\
(n=500)\end{array}$ & $\begin{array}{l}\text { Non-responders } \\
(\mathrm{n}=167)\end{array}$ & p Value & $\begin{array}{l}\text { CR group } \\
(n=277)\end{array}$ & $\begin{array}{l}\text { No CR group } \\
(n=223)\end{array}$ & p Value \\
\hline Age & $68.4(12.5)$ & $69.2(12.0)$ & $66.0(13.6)$ & $<0.01$ & $68.2(11.9)$ & $70.5(12.0)$ & 0.04 \\
\hline Male & $423(63.4)$ & $327(65.4)$ & $96(57.5)$ & 0.07 & $186(67.2)$ & $141(63.2)$ & 0.35 \\
\hline Married & $411(61.6)$ & $323(64.6)$ & $88(52.7)$ & $<0.01$ & $195(70.4)$ & $128(57.4)$ & 0.02 \\
\hline \multicolumn{8}{|l|}{ Labour market attachment } \\
\hline Employed & $152(22.8)$ & $116(23.2)$ & $36(21.6)$ & \multirow{3}{*}{$<0.01$} & $65(23.5)$ & $51(22.9)$ & \multirow{3}{*}{0.61} \\
\hline Unemployed & $14(2.1)$ & $6(1.2)$ & $8(4.8)$ & & $5(1.8)$ & $1(0.4)$ & \\
\hline Retired & $445(69.7)$ & $344(68.8)$ & $101(60.5)$ & & $191(69.0)$ & $153(68.6)$ & \\
\hline \multicolumn{8}{|l|}{ Educational level } \\
\hline Maximum of 9 school years & $277(41.5)$ & $207(41.4)$ & $70(41.9)$ & \multirow{4}{*}{0.09} & $114(41.2)$ & $93(41.7)$ & \multirow{4}{*}{0.70} \\
\hline High school, vocational training & $264(39.6)$ & $200(40.0)$ & $64(38.3)$ & & $112(40.4)$ & $88(39.5)$ & \\
\hline University degree & $103(15.4)$ & $77(15.4)$ & $26(15.6)$ & & $48(17.3)$ & $29(13.0)$ & \\
\hline Missing & $23(3.4)$ & $16(3.2)$ & 7 (4.2) & & $3(1.1)$ & $13(5.8)$ & \\
\hline \multicolumn{8}{|l|}{ Annual income $(€)$} \\
\hline 0-20000 & $295(44.2)$ & $206(41.2)$ & $89(53.3)$ & \multirow{3}{*}{$<0.01$} & $102(36.8)$ & $104(46.6)$ & \multirow{3}{*}{0.09} \\
\hline $20000-30000$ & 209 (31.3) & $160(32.0)$ & $49(29.3)$ & & $95(34.3)$ & $65(29.1)$ & \\
\hline$\geq 30000$ & $163(24.4)$ & $134(26.8)$ & $29(17.4)$ & & $80(28.9)$ & $54(24.2)$ & \\
\hline \multicolumn{8}{|l|}{ Type of surgery } \\
\hline Aortic valve & $519(77.5)$ & $392(78.4)$ & $127(76.0)$ & 0.54 & $208(75,1)$ & $184(82.5)$ & 0.04 \\
\hline Mitral valve & $159(25.2)$ & $123(24.6)$ & $36(21.6)$ & 0.41 & $74(26.7)$ & $49(22.0)$ & 0.22 \\
\hline Tricuspid valve & $10(1.5)$ & $7(1.4)$ & $3(1.8)$ & 0.72 & $5(1.8)$ & $2(0.9)$ & 0.39 \\
\hline Pulmonal valve & $11(1.6)$ & $6(1.2)$ & $5(3.0)$ & 0.11 & $4(1.4)$ & $2(0.9)$ & 0.58 \\
\hline Concomitant CABG & $203(31.3)$ & $152(30.4)$ & $51(30.5)$ & 0.91 & $89(32.1)$ & $63(28.3)$ & 0.31 \\
\hline \multicolumn{8}{|l|}{ Cardiac history } \\
\hline Previous heart valve surgery & $4(0.6)$ & $3(0.6)$ & $1(0.6)$ & 0.92 & $2(0.7)$ & $1(0.4)$ & 0.69 \\
\hline Previous CABG & $20(3.0)$ & $13(2.6)$ & $7(4.2)$ & 0.30 & $7(2.5)$ & $6(2.7)$ & 0.91 \\
\hline Previous MI & $83(12.4)$ & $51(10.2)$ & $32(19.2)$ & $<0.01$ & $29(10.5)$ & $22(9.9)$ & 0.78 \\
\hline Heart failure & $136(20.4)$ & $92(18.4)$ & $44(26.3)$ & 0.02 & $46(16.6)$ & $46(20.6)$ & 0.21 \\
\hline Cerebrovascular disease & $59(8.8)$ & $43(8.6)$ & $16(9.6)$ & 0.71 & $28(10.1)$ & $15(6.7)$ & 0.17 \\
\hline Arrhythmia & $226(33.9)$ & $173(34.6)$ & $53(31.7)$ & 0.70 & $105(37.9)$ & $68(30.5)$ & 0.08 \\
\hline \multicolumn{8}{|l|}{ Comorbidity } \\
\hline Renal Disease & $32(4.8)$ & $23(4.6)$ & $9(5.4)$ & 0.74 & $15(5.4)$ & $8(3.6)$ & 0.74 \\
\hline COPD & $68(10.2)$ & $42(8.4)$ & $26(15.6)$ & $<0.001$ & $27(9.7)$ & $15(6.7)$ & 0.16 \\
\hline Diabetes mellitus & $74(11.1)$ & $53(10.6)$ & $21(12.6)$ & 0.53 & $26(9.4)$ & $27(12.1)$ & 0.32 \\
\hline Charlson comorbidity index score & $1.0(1.4)$ & $0.9(1.3)$ & $1.3(1.6)$ & $<0.01$ & 0.9 & 0.9 & 0.92 \\
\hline
\end{tabular}

Values are number of patients (\%) for categorical variables and mean (SD) for continuous variables.

$p$ Values are derived from $\chi^{2}$ test for categorical variables and student's independent samples t test for continuous variables.

CABG, coronary artery bypass graft; COPD, chronic obstructive pulmonary disease; $\mathrm{CR}$, cardiac rehabilitation; MI, myocardial infarction 
Table 2 Healthcare resource utilisation and sick leave 12 months before and after exercise-based cardiac rehabilitation following heart valve surgery

\section{CR group ( $n=277)$} Before

After

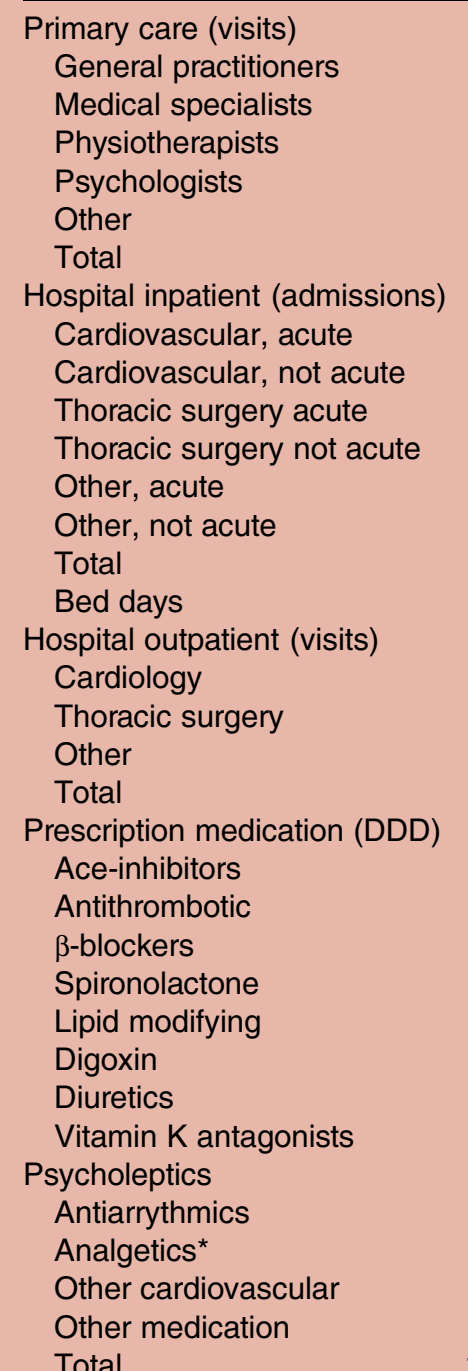

Sick leave (weeks)
$15.7(0.7)$

$0.5(0.0)$

$0.1(0.1)$

$0.0(0.0)$

$0.8(0.0)$

$17.2(0.7)$

$0.6(0.1)$

$0.5(0.0)$

$0.1(0.0)$

$1.0(0.0)$

$1.1(0.1)$

$0.4(0.0)$

$3.6(0.1)$

$21.9(1.1)$

$3.0(0.2)$

$0.2(0.1)$

$6.7(0.7)$

$9.9(0.8)$

$145.6(17.9)$

$175.2(12.0)$

$51.7(5.5)$

6.5 (1.9)

$62.4(13.5)$

12.5 (3.7)

$271.2(62.1)$

$31.8(4.1)$

$0.5(0.5)$

$7.1(2.0)$

$32.6(5.4)$

$238.5(20.8)$

$553.4(32.8)$

$1588.6(99.9)$
$25.3(0.9)$

$0.5(0.0)$

$0.1(0.0)$

$0.0(0.0)$

$0.8(0.0)$

$26.7(0.9)$

$0.3(0.1)$

$0.1(0.0)$

$0.0(0.0)$

$0.0(0.0)$

$0.7(0.1)$

$0.2(0.0)$

$1.4(0.1)$

$5.9(0.8)$

$5.3(0.6)$

$1.3(0.2)$

$12.6(0.9)$

$19.3(1.0)$

248.9 (25.2)

$132.2(8.1)$

14.7 (2.7)

$115.9(18.8)$

$16.2(3.5)$

350.8 (37.8)

$124.8(8.7)$

$0.5(0.5)$

$33.5(5.0)$

$46.6(6.2)$

254.2 (21.9)

704.2 (33.0)

$2310.3(80.2)$
267.8 (14.2)

Difference

\section{No CR group $(n=223)$}

Before

$9.6(0.8)$

$0.0(0.0)$

$0.0(0.0)$

$0.0(0.0)$

$0.0(0.0)$

$9.5(0.9)$

$-0.2(0.1)$

$-0.4(0.0)$

$-0.1(0.0)$

$-1.0(0.0)$

$-0.4(0.1)$

$-0.1(0.1)$

$-2.2(0.1)$

$-15.9(1.1)$

$2.4(0.6)$

$1.1(0.2)$

$6.0(0.7)$

$9.4(0.9)$

$103.3(22.2)$

$92.6(14.8)$

$80.5(7.8)$

$8.2(2.5)$

53.5 (13.6)

$3.6(4.6)$

$79.5(66.0)$

$93.0(8.7)$

$0.00(0.0)$

$26.3(4.7)$

$14.4(3.0)$

15.7 (22.8)

150.8 (23.2)

721.7 (96.6)

$3.4(0.6)$

$17.1(0.8)$

$0.5(0.3)$

$0.1(0.0)$

$0.0(0.0)$

$0.8(0.0)$

$18.5(0.8)$

$0.6(0.1)$

$0.5(0.0)$

$0.1(0.0)$

$1.0(0.0)$

$1.4(0.1)$

$0.3(0.0)$

$4.0(0.2)$

24.3 (1.5)

$2.5(0.2)$

$0.3(0.0)$

$6.0(0.4)$

$8.8(0.5)$

148.1 (19.5)

158.9 (13.2)

$53.4(6.2)$

$8.3(2.1)$

$59.3(16.7)$

$11.4(3.5)$

219.3 (51.9)

$34.2(5.1)$

$9.1(1.1)$

$47.2(7.8)$

238.8 (25.4)

$609.1(40.9)$

$1599.0(100.0)$
$1.8(0.9)$

After

$27.1(1.1)$

$0.5(0.5)$

$0.1(0.0)$

$0.0(0.0)$

$0.8(0.0)$

$28.7(1.1)$

$0.4(0.1)$

$0.1(0.0)$

$0.1(0.0)$

$0.0(0.0)$

$1.0(0.1)$

$0.2(0.0)$

$1.8(0.2)$

$7.2(1.0)$

$3.8(0.5)$

$0.7(0.1)$

$8.2(0.9)$

$12.7(1.1)$

222.9 (25.8)

263.9 (14.0)

113.7 (8.9)

17.9 (3.4)

118.7 (24.9)

18.8 (3.9)

344.8 (30.8)

$112.0(8.8)$

$1.8(0.9)$

$26.7(5.2)$

$67.0(8.5)$

$241.2(22.8)$

817.5 (47.4)

$2367.0(93.2)$

$3.5(0.7)$

$1.2(0.3)$
Values are mean number of visits/amounts and associated SE 1 year presurgery and postsurgery.

*Includes analgetics and topical products with DDD value for joint and muscular pain.

$\mathrm{CR}$, cardiac rehabilitation, DDD, defined daily doses.

Difference

Difference between

group difference

p Value

$10.1(0.9)$

$0.0(0.3)$

$0.0(0.0)$

$0.0(0.0)$

$0.0(0.0)$

$10.2(0.9)$

$-0.1(0.1)$

$-0.4(0.0)$

$0.0(0.0)$

$-1.0(0.0)$

$-0.4(0.2)$

$-0.2(0.1)$

$-2.2(0.2)$

$-17.1(1.7)$

$1.3(0.5)$

$0.3(0.1)$

$2.2(0.8)$

$3.9(1.0)$

$0.5(-2.9$ to 1.9$)$

$0.0(-0.1$ to 0.0$)$

$0.0(0.0$ to 0.0$)$

0.0 (0.0 to 0.0$)$

$0.0(0.0$ to 0.1$)$

$-0.6(-3.1$ to 1.8$)$

$-0.1(-0.3$ to 0.1$)$

0.1 (-0.1 to 0.2$)$

$-0.1(-0.1$ to 0.0$)$

$0.0(-0.1$ to 0.1$)$

$-0.1(-0.4$ to 0.3$)$

$0.1(-0.1$ to 0.2$)$

$-0.1(-0.6$ to 0.4$)$

$1.2(-2.9$ to 5.2$)$

$1.0(-0.5$ to 2.6$)$

0.8 (0.3 to 1.3$)$

$3.8(1.6$ to 5.9$)$

5.6 (2.9 to 8.3$)$

74.8 (22.6) $\quad 28.4(-31.7$ to 88.6$)$

$105.0(16.4) \quad-12.4(-55.7$ to 31.0$)$

$60.3(8.0) \quad 20.2(-1.6$ to 42.1$)$

$9.6(3.4) \quad-1.4(-9.4$ to 6.8$)$

$59.4(18.6) \quad-5.9(-50.4$ to 38.7$)$

$7.3(3.8)$

$-3.7(-15.5$ to 8.0$)$

$125.5(52.0) \quad-46.0(-211.2$ to 119.3$) 0.58$

$77.8(8.8) \quad 15.2(-9.1$ to 39.6$)$

$0.0(0.0)$

$0.0(-2.5$ to 2.5$)$

$17.6(5.6)$

$8.7(-5.8$ to 23.2$)$

$-5.4(-18.0$ to 7.2$)$

$23(21.4) \quad 13.4(-47.5$ to 74.3$)$

$208.4(27.8) \quad-57.6(-128.1$ to 12.9$)$

$768.0(78.2)$

$-46.2(-287.2$ to 195.5$)$

$2.4(0.5)$

$1.0(-0.6$ to 2.7$)$

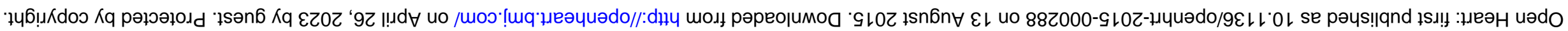


Table 3 Costs of care 12 months before and after exercise-based cardiac rehabilitation following heart valve surgery

\begin{tabular}{|c|c|c|c|c|c|c|c|c|}
\hline & \multicolumn{3}{|c|}{ CR group $(n=277)$} & \multicolumn{3}{|c|}{ No CR group $(n=223)$} & \multirow{2}{*}{$\begin{array}{l}\text { Difference between } \\
\text { group difference }\end{array}$} & \multirow[b]{2}{*}{ p-Value } \\
\hline & Before & After & Difference & Before & After & Difference & & \\
\hline \multicolumn{9}{|l|}{ Primary care (visits) } \\
\hline General practitioners & 337 (17) & $544(23)$ & 207 (22) & $352(17)$ & $569(25)$ & $217(22)$ & $-10.0(-70$ to 50$)$ & 0.74 \\
\hline Medical specialists & $153(21)$ & $113(14)$ & $-40(18)$ & $136(17)$ & $125(16)$ & $-11(23)$ & $-29(-87$ to 28$)$ & 0.31 \\
\hline Physiotherapists & $26(8)$ & $26(10)$ & $0(0)$ & $51(25)$ & $38(16)$ & $-13(17)$ & $13(-22$ to 48$)$ & 0.46 \\
\hline Psychologists & $3(2)$ & $8(4)$ & $5(5)$ & $2(1)$ & $6(3)$ & $4(3)$ & $1(-10$ to 11$)$ & 0.93 \\
\hline Other & $93(6)$ & $81(6)$ & $-12(6)$ & $83(6)$ & $78(6)$ & $-5(7)$ & $-7(-24$ to 11$)$ & 047 \\
\hline Total & $612(30)$ & $773(32)$ & $161(32)$ & $624(41)$ & $817(35)$ & $193(37)$ & $-32(-126$ to 61$)$ & 0.51 \\
\hline \multicolumn{9}{|c|}{ Hospital inpatient (admissions) } \\
\hline Cardiovascular, acute & $3655(814)$ & $1656(392)$ & $-1999(828)$ & $3532(914)$ & $1862(312)$ & $-1670(897)$ & $-329(-2732$ to 2072$)$ & 0.78 \\
\hline Cardiovascular, not acute & $1438(151)$ & $675(243)$ & $-763(273)$ & $1931(308)$ & $848(294)$ & $-1083(399)$ & $320(-616$ to 1258$)$ & 0.51 \\
\hline Thoracic surgery, acute & $3798(813)$ & $469(314)$ & $-3329(837)$ & $2950(818)$ & $446(196)$ & $-2504(847)$ & $-825(-3158$ to 1508$)$ & 0.48 \\
\hline Thoracic surgery, not acute & $28902(904)$ & $251(136)$ & $-28651(913)$ & 28091 (839) & $177(145)$ & -27914 (839) & $-737(-3187$ to 1716$)$ & 0.56 \\
\hline Other, acute & $5143(563)$ & 2682 (394) & $2461(602)$ & $6649(899)$ & $3904(517)$ & $-2745(1007)$ & $284(-2017$ to 2585$)$ & 0.81 \\
\hline Other, not acute & $1456(225)$ & $1314(236)$ & $-142(317)$ & $2111(724)$ & 705 (186) & $-1406(757)$ & 1264 (-358 to 2883$)$ & 0.13 \\
\hline Total & $44391(1806)$ & 7048 (879) & $-37343(1766)$ & 45264 (2173) & $7942(847)$ & $-31321(2173)$ & $-22(-5451$ to 5406$)$ & 0.99 \\
\hline \multicolumn{9}{|l|}{ Hospital outpatient (visits) } \\
\hline Cardiology & $730(59)$ & 909 (101) & $179(114)$ & $658(57)$ & $610(78)$ & $-48(86)$ & 227 ( -47 to 503$)$ & 0.11 \\
\hline Thoracic surgery & $33(8)$ & $184(28)$ & $151(27)$ & $43(8)$ & $84(17)$ & $41(19)$ & 110 (46 to 177$)$ & $<0.01$ \\
\hline Other & $1945(265)$ & 2147 (303) & 202 (177) & $1734(121)$ & $1868(365)$ & $134(333)$ & $68(-672$ to 807$)$ & 0.86 \\
\hline Total & $2707(273)$ & $3240(315)$ & 533 (209) & 2435 (139) & $2561(376)$ & $126(342)$ & $407(-378$ to 1191$)$ & 0.29 \\
\hline \multicolumn{9}{|c|}{ Pharmaceuticals (drug categories) } \\
\hline ACE-inhibitors & $9(1.2)$ & $11(1)$ & $2(1)$ & $11(2)$ & $12(2)$ & $1(1)$ & $2(-1$ to 6$)$ & 0.23 \\
\hline Antithrombotic & $29(3)$ & $59(18)$ & $30(18)$ & $29(2)$ & $34(5)$ & $5(8)$ & $25(-14$ to 65$)$ & 0.21 \\
\hline$\beta$-blockers & $15(2)$ & $31(2)$ & $16(2)$ & $13(2)$ & $27(3)$ & $14(3)$ & $2(-4$ to 9$)$ & 0.47 \\
\hline Spironolactone & $2(1)$ & $5(1)$ & $3(1)$ & $3(1)$ & $6(1)$ & $3(1)$ & $0(-3$ to 2$)$ & 0.68 \\
\hline Lipid modifying & $57(12)$ & $86(13)$ & $29(9)$ & $44(12)$ & $69(14)$ & $25(10)$ & $4(-23$ to 30$)$ & 0.78 \\
\hline Digoxin & $2(1)$ & $3(1)$ & $1(1)$ & $2(1)$ & $4(1)$ & $2(1)$ & $-1(-4$ to 1$)$ & 0.21 \\
\hline Diuretics & $24(4)$ & $25(2)$ & $1(4)$ & $24(3)$ & $25(2)$ & $1(3)$ & $0(-10$ to 10$)$ & 0.99 \\
\hline Vitamin $\mathrm{K}$ antagonists & $14(2)$ & $60(6)$ & $46(6)$ & $17(2)$ & $56(7)$ & $39(6)$ & $7(-11$ to 25$)$ & 0.45 \\
\hline Psycholeptics & $0(0)$ & $0(0)$ & $0(0)$ & $5(4)$ & $11(5)$ & $6(4)$ & -6 ( -13 to 2$)$ & 0.12 \\
\hline Antiarrythmics & $2(0)$ & $6(1)$ & $4(1)$ & $3(1)$ & $6(1)$ & $3(1)$ & $1(-2$ to 4$)$ & 0.36 \\
\hline Analgetics* & $13(3)$ & $16(3)$ & $3(2)$ & $28(8)$ & $29(8)$ & $1(6)$ & $2(-10$ to 13$)$ & 0.76 \\
\hline Other cardiovascular & $43(6.3)$ & $25(4)$ & $-18(5)$ & $57(8)$ & $24(4)$ & $-33(7)$ & $15(-2$ to 33$)$ & 0.09 \\
\hline Other medication & $256(26)$ & $254(24)$ & $-2(15)$ & $305(35)$ & $300(28)$ & $-5(23)$ & $3(-51$ to 57$)$ & 0.91 \\
\hline Total & $466(34)$ & $581(35)$ & $115(30)$ & $541(46)$ & $602(39)$ & $61(30)$ & $54(-28$ to 136$)$ & 0.88 \\
\hline Total cost (healthcare) & $48176(1897)$ & $11641(960)$ & $-36535(1786)$ & $48864(1786)$ & $11922(1040)$ & $-36943(2225)$ & $408(-5083$ to 5896$)$ & 0.89 \\
\hline Sick leave, weeks & $1320(278)$ & $4424(681)$ & 3104 (561) & $1079(256)$ & $3260(645)$ & $2181(503)$ & $923(-579$ to 2426$)$ & 0.23 \\
\hline Total costs (societal) & 49496 (1965) & $16065(1191)$ & $-33431(1925)$ & $49943(2334)$ & $15182(1269)$ & $-34761(2290)$ & $1330(-4427$ to 7086$)$ & 0.65 \\
\hline
\end{tabular}



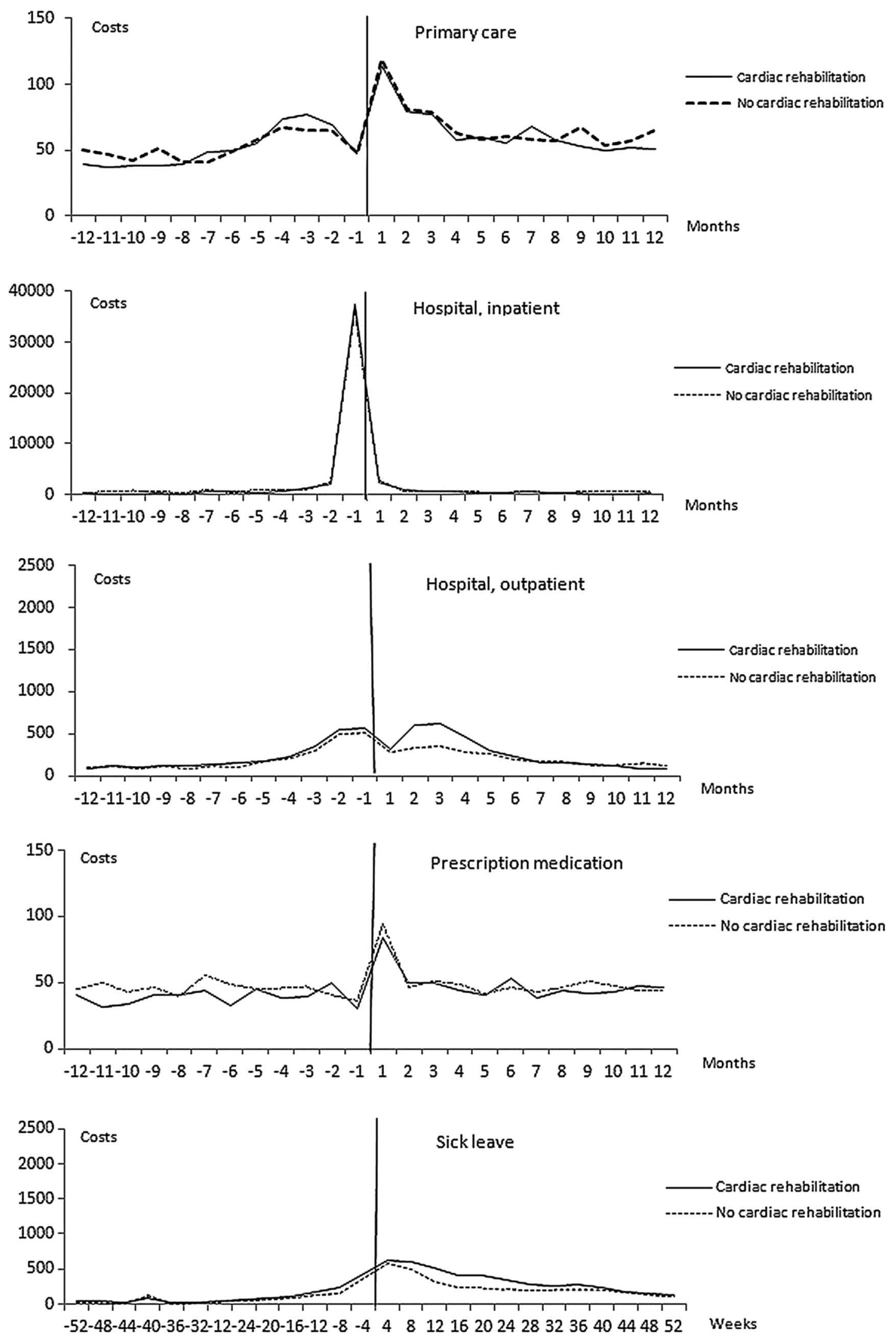

Figure 1 Cost of healthcare and sick leave before and after heart valve surgery $(€)$. Monthly costs of healthcare and sick leave 1 year before and after heart valve surgery. Owing to the current weekly register practice. Sick leave costs are presented as 4-week months. Time 0 indicates time of surgery. Values are mean values with $95 \%$ bootstrapped Cls. 
healthcare utilisation, sick leave or costs for any of the other categories of primary care, hospital inpatient admissions, prescription medication or sick leave, between the groups. We also found no statistically significant differences in either total healthcare use or total costs $€ 1330 \quad(-4427$ to $7086, \mathrm{p}=0.65)$ between the groups.

Although non-significant, some tendencies were found, including reductions in acute inpatient hospital admissions per patient in cardiovascular, thoracic surgery and other departments, in favour of the CR group. We also found a non-significant higher number of sick leave weeks and costs per patient in the CR group (table 2).

The total costs per patient for the year before heart valve surgery were estimated at $€ 49496$ (45645 to 53 346) per patient in the CR group and $€ 49943$ (45 367 to 54517 ) in the no CR group and, respectively, $€ 16065$ (13 730 to 18399$)$ and €15 182 (12 695 to 17670 ) for the year following surgery (table 3 ). The main cost driver during the presurgery period was the heart valve surgery index hospital admission, and hospital inpatient admissions postsurgery. In both groups, an increase in costs per patient was found overall for primary healthcare visits, outpatient visits, prescription medication and sick leave from the presurgery to postsurgery periods, while a decrease was found across hospital inpatient admission categories.

Figure 1 illustrates the monthly distribution of costs. Following surgery, for both groups, the costs associated with primary care visits, prescription medication and sick leave, peaked during the first month following discharge and gradually returned to a level corresponding to the 12 months before surgery by the end of follow-up. Also, costs due to inpatient admissions were highest within the first month postsurgery. Patients were admitted during the first month due to primary diagnoses, ranging from heart failure, arrhythmia, infective endocarditis, other infections, cancer, stroke, myocardial infarction and pericardial effusion, to allergic reactions. Decreases in outpatient visits and costs were observed immediately after surgery, followed by an increase for the CR group, corresponding to the CR participation period.

Owing to baseline differences between responders and non-responders, we analysed the differences in presurgery to postsurgery costs for non-responders $(n=167)$ for the categories of primary care visits $€ 242$ (146 to 337 ), outpatient visits $€ 265$ (-297 to 827), hospital inpatient admissions $€-37147$ (-42 221 to -32071 ), prescription medication $€ 94$ ( -19 to 206$)$, sick leave 1835 (484 to 3186) and total costs $€-37711$ (-40 102 to -29 321) for comparison with the CR and no CR groups (data not shown).

\section{DISCUSSION}

This study investigated the cost consequences of CR in patients undergoing heart valve surgery from a broad analytical perspective. Overall, we found that provision of CR appears to be cost neutral, with no differences in total costs of healthcare or sick leave. This finding masked a higher number of outpatient visits among CR participants, which could only be partly explained by CR participation, indicating that CR patients potentially substitute more expensive outpatient visits with the rehabilitation services offered.

No economic evaluations or cost analyses have previously investigated the economic implications of CR to heart valve surgery patients. Difficulties comparing costs across patient populations were highlighted in a systematic review reporting a range of costs estimated as $€ 1,940-€ 24,941$ per life year gained across economic evaluations included. ${ }^{13}$ Among three studies conducted alongside randomized trials and included in a recent Cochrane review on the effect of CR to coronary artery disease patients, two studies found no statistically significant differences in total health care costs between CR participants and usual care, while the third reported higher total healthcare costs associated with CR (EUR 4,280.97 more per patient). ${ }^{25}$ All studies reported limited data on costs per patient and could not be compared due to different timing and currency and are not directly comparable to our study.

Also, two cost analyses have been conducted as part of non-randomized studies among coronary artery disease patients with 21 months and 5 years of follow-up. ${ }^{26} 27$ Data from these are not directly comparable to our study due to timing of the studies. However, CR participation reduced cardiovascular hospital admissions in both studies, and in the 5-year follow-up study, CR participants returned to work more frequently.

In our study, we found no corresponding impact of CR on inpatient hospital admissions or costs even though hospital inpatient admissions were cost drivers following surgery, which may have several explanations. Hospital admissions to cardiovascular departments were second to acute admissions to other departments, indicating that these patients have other health problems not influenced by CR. Additionally, the majority of hospital admissions were observed during the first month postsurgery with the majority considered nonpreventable by CR. The difference in recurrent cardiac events and readmissions observed for ischaemic heart disease patients during long-term follow-up may be partly explained by the effect of CR on risk factors, whereas a proportion of heart valve surgery patients do not have concomitant ischaemic heart disease. Economic incentive models have recently been implemented in some healthcare systems, where readmissions after cardiac events are used as determinants of reimbursement depending on CR participation within 1 month following cardiac events. ${ }^{28}{ }^{29}$ Such models may not be suitable to heart valve surgery patients.

The difference in recurrent cardiac events and readmissions observed for ischemic heart disease patients in the two cost analyses compared to our study during long- 
term follow-up may be partly explained by the effect of CR programmes on risk factors, where a proportion of heart valve surgery patients do not have concomitant ischemic heart disease. Therefore other outcome measures such as HRQL may be an important outcome measure when evaluating CR to heart valve surgery patients, particularly since HRQL has been shown to be below the level of general population up to 6-12 months post surgery. ${ }^{30}$ Also, heart valve surgery patients without ischemic heart disease may benefit from a specially targeted programme potentially including less components and therefore less costly than for patients with ischemic heart disease. We found that, while all patients in our sample participated in physical exercise training, a varying proportion of patients in clinical practice received other components of the CR programme, as recommended. ${ }^{7817} \mathrm{An}$ area for future research would be to evaluate different CR programmes aimed at these patients. In Denmark, part of the CR programme (eg, physical exercise training) can be conducted in cardiovascular outpatient hospital settings, but also in other medical departments or departments of thoracic surgery, which explains why the increase in visits from presurgery to postsurgery associated with CR participation was not solely reflected in cardiovascular outpatient visits. The number of outpatient visits due to CR in our study was much lower than expected given current recommendations and partly reflects lack of information on the number of visits conducted in municipalities, albeit the majority of patients participated in a shared or full hospital-based CR programme. However, low adherence has been reported formerly. In a recent Danish study, conducted at a large Danish university hospital, of 266 cardiac surgery patients, $112(42 \%)$ did not complete CR, consisting of a 8-week course with 1-h biweekly sessions, with non-completion defined as attendance $\leq 75 \%$ of sessions, 80 patients (30\%) completed all sessions, and 74 patients (28\%) referred to CR never joined. A quarter of all patients were readmitted within 8 weeks post surgery, and readmission was found to be the strongest predictor of non-completion CR. ${ }^{31}$ Compared to our study, data on CR attendance was recorded in a database, indicating a high participation rate but low CR adherence among these patients in Denmark due to particularly high readmission rates. Even higher readmission rates were subsequently reported in Denmark following heart valve surgery specifically $(\mathrm{n}=867)$ with $26 \%$ of the population readmitted within 30 days and $56 \%$ having at least one readmission during 12 months follow-up post discharge. ${ }^{30}$ Higher CR attendance might potentially have increased the costs for the CR group and/ or have substituted costs in other cost categories and thereby change the overall findings. An important area for future research is to compare CR programmes of different duration and intensity for this group of patients from both a clinical and an economic perspective.

Although the difference was not significant, in our study, CR participants were on sick leave for a longer period compared to non-participants, incurring higher costs. In some studies, faster return to work among cardiac patients enrolled in a CR programme has been demonstrated. ${ }^{10}{ }^{32}$ A French study including patients after acute ST-segment elevation myocardial infarction found no effect of CR on return to work and, additionally, CR participants returned to work later than nonparticipants. ${ }^{11}$ We included a nationwide cohort of patients with only a marginal difference in the employment rates of responders and non-responders, and in which a high number of patients had retired by the time of follow-up. The low employment rate illustrates that sick leave and return to work may not be important outcomes in CR studies among heart valve surgery patients. However, looking at the effect of CR among work-active cardiac patients would be relevant to investigate if CR participation contributes to patients remaining on sick leave longer than expected.

We included heart valve surgery patients broadly, even though healthcare utilisation, complications and costs of care have been shown to differ across type of surgery. ${ }^{33}$ The difficulties of implementing CR recommendations in clinical practice highlight the importance of supplementing the results of randomised trials with economic data from well-performed observational studies conducted from a broad perspective. Our study provides high external validity and illustrates what happens in a real-life setting without exclusion criteria, with national variation in content and cost of $\mathrm{CR}$ provision, and imperfect CR uptake and adherence, based on wide CR recommendations. However, this study provides comparably lower internal validity due to less detailed patientlevel information on CR services received.

Until further studies are conducted, CR can be provided to heart valve surgery patients based on recommendations for patients with ischaemic heart disease, since it imposes no significant additional costs to a national healthcare system. However, we recommend that studies of the benefits of CR on patient-reported outcomes, for example, HRQL, preferably as part of a formal economic evaluation, be conducted to provide evidence for or against CR provision, specifically to heart valve surgery patients, from an economic perspective. Additionally, a proportion of patients without concomitant ischaemic heart disease may need a specialised CR programme.

\section{Strengths and limitations}

The main strength is that we included a national cohort of consecutive unselected patients with minor baseline differences between CR participants versus nonparticipants and complete follow-up. Owing to the minor baseline differences, a difference-in-difference design was employed to overcome the limitation that our study was not randomised. However, residual confounding factors cannot be definitely excluded. On the other hand, unlike an unblinded experiment, this study design has not influenced the behaviour of the patients, including healthcare utilisation. 
Another strength is the high survey response rate. Owing to baseline differences between responders and non-responders, we compared presurgery and postsurgery healthcare use and costs across non-responders, CR participants and non-participants. For non-responders compared to the other groups, for sick leave, the cost difference from presurgery to postsurgery was smaller and, for primary care, the difference was larger. No differences were observed across the remaining cost categories. However, the lack of self-reported information on CR participation among non-responders is important to consider when addressing generalisability.

Our study has limitations. The major limitation in this study is, that we do not have exact detailed information about the duration of the CR programmes and number of sessions attended since this information could not be obtained from self-reported data due to the risk of recall bias using a retrospective design, and also complete and valid register based data on municipality-based CR programmes are not available in Denmark. Additionally, our study could not be reported as a cost-effectiveness/ cost-utility study, since we did not have clinical or patient-reported quality of life outcomes for the groups compared.

Also, register data are subject to bias due to misclassifications and different definitions used over time. The validity of our study is considered high due to the short period of two years. Finally, we did not have information on sick leave within the first two weeks following heart valve surgery.

\section{CONCLUSIONS}

CR, as provided in Denmark, after heart valve surgery, can be considered cost neutral. CR participation is associated with more outpatient visits but may reduce other more expensive outpatient visits. Further studies should investigate the benefits of CR on heart valve surgery patient-reported outcomes, for example, HRQL, as part of a formal cost-utility analysis.

\section{Author affiliations \\ ${ }^{1}$ Department of Cardiology, Roskilde Hospital, Roskilde, Denmark \\ ${ }^{2}$ Centre for Applied Health Services Research, University of Southern Denmark, Odense, Denmark \\ ${ }^{3}$ Department of Cardiology, The Heart Centre, Rigshospitalet, Copenhagen University Hospital, Copenhagen, Denmark. \\ ${ }^{4}$ National Institute of Public Health, University of Southern Denmark, Copenhagen, Denmark \\ ${ }^{5}$ National Centre of Rehabilitation and Palliation, University of Southern Denmark and University Hospital of Odense, Odense, Denmark ${ }^{6}$ Department of Health Sciences, University of York, York, UK ${ }^{7}$ Department of Public Health, Aarhus University, Aarhus, Denmark ${ }^{8}$ Department of Clinical Medicine, Aarhus University, Aarhus, Denmark}

Funding This study was supported by the Danish Strategic Research Council (grant number: 10-092790) and the Region Zealand, Denmark.

Competing interests None declared.

Provenance and peer review Not commissioned; externally peer reviewed.

Data sharing statement Additional unpublished data are available to the research team of the CopenHeart project group.
Open Access This is an Open Access article distributed in accordance with the Creative Commons Attribution Non Commercial (CC BY-NC 4.0) license, which permits others to distribute, remix, adapt, build upon this work noncommercially, and license their derivative works on different terms, provided the original work is properly cited and the use is non-commercial. See: http:// creativecommons.org/licenses/by-nc/4.0/

\section{REFERENCES}

1. Nkomo VT, Gardin JM, Skelton TN, et al. Burden of valvular heart diseases: a population-based study. Lancet 2006;368:1005-11.

2. LaPar DJ, Crosby IK, Rich JB, et al. A contemporary analysis of postoperative morbidity after coronary artery bypass grafting with and without concomitant aortic valve replacement to improve patient quality and cost-effective care. Ann Thorac Surg 2013;96:1621-7.

3. Lee R, Li S, Rankin JS, et al. Fifteen-year outcome trends for valve surgery in North America. Ann Thorac Surg 2011;91:677-84.

4. Shan L, Saxena A, McMahon R, et al. A systematic review on the quality of life benefits after aortic valve replacement in the elderly. $J$ Thorac Cardiovasc Surg 2013;145:1173-89.

5. Berg SK, Zwisler AD, Pedersen BP, et al. Patient experiences of recovery after heart valve replacement: suffering weakness, struggling to resume normality. BMC Nurs 2013;12:23.

6. Sibilitz KL, Berg SK, Tang LH, et al. Exercise-based cardiac rehabilitation for adults after heart valve surgery. Cochrane Database Syst Rev, in press.

7. Butchart EG, Gohlke-Barwolf C, Antunes MJ, et al. Recommendations for the management of patients after heart valve surgery. Eur Heart J 2005;26:2463-71.

8. Danish Health and Medicines Authority. Pakkeforløb for hjerteklapsygdom og hjertesvigt. [Recommendations for heart valve surgery and heart failure patients: pathways through the health care system]. Copenhagen: Danish Health and Medicines Authority, 2009.

9. Andersson LJ, Taylor RS. Cardiac rehabilitation for people with heart disease: an overview of Cochrane systematic reviews. Int J Cardiol 2014;177:348-61.

10. Fonager K, Lundbye-Christensen S, Jesper Andreasen JJ, et al. Work status and return to the workforce after coronary artery bypass grafting and/or heart valve surgery: a one-year-follow up study. Rehabil Res Pract 2014;2014:631842.

11. Isaaz K, Coudrot M, Sabry MH, et al. Return to work after acute ST-segment elevation myocardial infarction in the modern era of reperfusion by direct percutaneous coronary intervention. Arch Cardiovasc Dis 2010;103:310-16.

12. Wong WP, Feng J, Pwee $\mathrm{KH}$, et al. A systematic review of economic evaluations of cardiac rehabilitation. BMC Health Serv Res 2012;12:243.

13. Papadakis S, Oldridge NB, Coyle D, et al. Economic evaluation of cardiac rehabilitation: a systematic review. Eur J Cardiovasc Prev Rehabil 2005;12:513-20.

14. Davies P, Taylor F, Beswick A, et al. Promoting uptake and adherence in cardiac rehabilitation. Cochrane Database Syst Rev 2010;(7):CD007131.

15. Lynge E, Sandegaard JL and Rebolj M. The Danish National Patient Register. Scand J Public Health 2011;39(suppl):30-3.

16. Würgler MW, Sonne LT, Kilsmark J, et al. Danish heart patients' participation in and experience with rehabilitation. Scand J Public Health 2012:40(suppl):126-32.

17. Danish Health and Medicines Authority. National klinisk retningslinje for hjerterehabilitering. [National clinical guidelines for cardiac rehabilitation]. Copenhagen: Danish Health and Medicines Authority, 2013.

18. Hansen TB, Berg SK, Sibilitz KL, et al. Availability of, referral to and participation in exercise-based cardiac rehabilitation after heart valve surgery: results from the national CopenHeart survey. Eur J Prev Cardiol 2015;22:710-18.

19. Charlson M, Pompei P, Ales K, et al. A new method of classifying prognostic comorbidity in longitudinal studies: development and validation. J Chronic Dis 1987;40:373-83.

20. Andersen JS, Olivarius NF, Krasnik A. The Danish National Health Service register. Scand J Public Health 2011;39(Suppl):34-7.

21. Wallach Kildemoes H, Toft Sørensen H, Hallas J. The Danish National Prescription Registry. Scand J Public Health 2011;39 (Suppl):38-41.

22. http://www.whocc.no/ddd/definition_and_general_considera/ (5 December 2014).

23. Stapelfeldt CM, Jensen C, Andersen NT, et al. Validation of sick leave measures: self-reported sick leave and sickness benefit data 
from a Danish national register compared to multiple workplace-registered sick leave spells in a Danish municipality. Validation of sick leave measures: self-reported sick leave and sickness benefit data from a Danish national register compared to multiple workplace-registered sick leave spells in a Danish municipality. BMC Public Health 2012;12:661.

24. Carpenter J, Bithell J. Bootstrap confidence intervals: when, which, what? A practical guide for medical statistics. Stat Med 2000;19:1141-65.

25. Heran BS, Chen JM, Ebrahim S. Exercise-based cardiac rehabilitation for coronary heart disease. Cochrane Database Syst Rev 2011 Jul 6;(7):CD001800. doi:10.1002/14651858.CD001800. pub2.

26. Levin LA, Perk J, Hedback B. Cardiac rehabilitation-a cost analysis. J Intern Med 1991;230:427-34.

27. Ades PA, Huang D, Weaver SO. Cardiac rehabilitation participation predicts lower rehospitalization costs. Am Heart J 1992;123 916-21.
28. Dunlay SM, Pack QR, Thomas RJ, et al. Participation in cardiac rehabilitation, readmission and death after acute myocardial infarction. Am J Med 2014;127:538-46.

29. Kaiser M, Varvel M, Doherty P. (2013) Making the case for cardiac rehabilitation: modelling potential impact on readmissions. http:// webarchive.nationalarchives.gov.uk/20130221101407/http://www. improvement.nhs.uk/documents/Case for CR.pdf

30. Sibilitz KL, Berg SK, Thygesen LC, et al. High readmission rate after heart valve surgery: A nationwide cohort study. Int $J$ Cardiol 2015;11:96-104.

31. Laustsen S, Hjortdal VE, Petersen AK. Predictors for not completing exercise-based rehabilitation following cardiac surgery. Scand Cardiovasc J 2013;47:344-51.

32. Sire S. Physical training and occupational rehabilitation after aortic valve replacement. Eur Heart J 1987;8:1215-20.

33. Bhamidipati CM, LaPar DJ, Fonner E Jr, et al. Outcomes and cost of cardiac surgery in octogenarians is related to type of operation: a multiinstitutional analysis. Ann Thorac Surg 2011;91:499-505. 